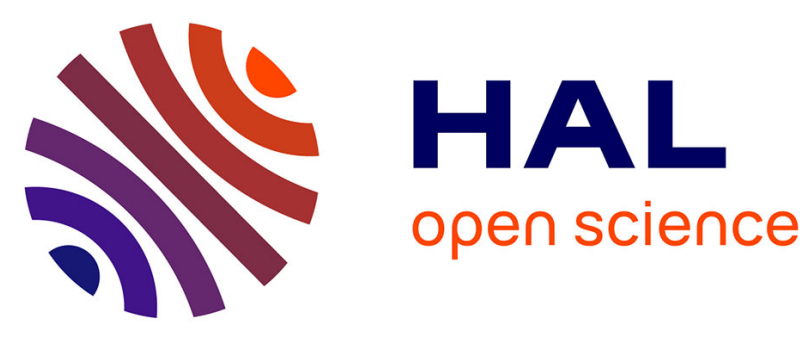

\title{
Prognostics of proton exchange membrane fuel cell stack in a particle filtering framework including characterization disturbances and voltage recovery.
}

Marine Jouin, Rafael Gouriveau, Daniel Hissel, Marie-Cécile Péra, Noureddine

Zerhouni

\section{To cite this version:}

Marine Jouin, Rafael Gouriveau, Daniel Hissel, Marie-Cécile Péra, Noureddine Zerhouni. Prognostics of proton exchange membrane fuel cell stack in a particle filtering framework including characterization disturbances and voltage recovery.. IEEE International Conference on Prognostics and Health Management, PHM'2014. - Enhancing Safety, Efficiency, Availability and Effectiveness of Systems through PHM Technology and Application., Jan 2014, United States. pp.1-6. hal-01050831

\section{HAL Id: hal-01050831 \\ https://hal.science/hal-01050831}

Submitted on 25 Jul 2014

HAL is a multi-disciplinary open access archive for the deposit and dissemination of scientific research documents, whether they are published or not. The documents may come from teaching and research institutions in France or abroad, or from public or private research centers.
L'archive ouverte pluridisciplinaire HAL, est destinée au dépôt et à la diffusion de documents scientifiques de niveau recherche, publiés ou non, émanant des établissements d'enseignement et de recherche français ou étrangers, des laboratoires publics ou privés. 


\title{
Prognostics of proton exchange membrane fuel cell stack in a particle filtering framework including characterization disturbances and voltage recovery
}

\author{
Marine Jouin, Rafael Gouriveau, Daniel Hissel, Marie-Cécile Péra and Noureddine Zerhouni \\ FEMTO-ST Institute (UMR CNRS 6174) - FCLAB (FR CNRS 3539) \\ Besançon, France \\ Email: firstname.lastname@femto-st.fr
}

\begin{abstract}
In the perspective of decreasing polluting emissions and developing alternative energies, fuel cells, and more precisely Proton Exchange Membrane Fuel Cells (PEMFC), represent a promising solution. Even if this technology is close to being competitive, it still suffers from too short life duration. As a consequence, prognostic seems to be a great solution to anticipate PEMFC stacks degradation. However, a PEMFC implies multiphysics and multiscale phenomena making the construction of an aging model only based on physics very complex. One solution consists in using a hybrid approach for prognostics combining the use of models and available data. Among these hybrid approaches, particle filtering methods seem to be really appropriate as they offer the possibility to compute models with time varying parameters and to update them all along the prognostics process. But to be efficient, not only should the prognostics system take into account the aging of the stack but also external events influencing this aging. Indeed, some acquisition techniques introduce disturbances in the fuel cell behavior and a voltage recovery can be observed at the end of the characterization process. This paper proposes to tackle this problem. First, PEMFC fuel cells and their complexities are introduced. Then, the impact of characterization of the fuel cell behavior is described. Empirical models are built and introduced in both learning and prediction phases of the prognostics model by combining three particle filters. The new prognostic framework is used to perform remaining useful life estimates and the whole proposition is illustrated with a long term experiment data set of a PEMFC in constant load solicitation and stable operating conditions. Estimates can be given with an error less than $5 \%$ for life durations of more than 1000 hours. Finally, the results are compared to a previous work to show that introducing a disturbance modeling can dramatically reduce the uncertainty coming with the predictions.
\end{abstract}

\section{INTRODUCTION}

Development of cleaner power sources is one of the major current challenges. Different kinds of fuel cells exist, made of different materials and using different fuel. In this study, we only focus on Proton Exchange Membrane Fuel Cell (PEMFC).

Although this technology is close from being competitive, it still suffers from a too short life duration impeding a large scale deployment. Extending this life duration can be achieved by several ways, one of them is Prognostics and Health Management(PHM). PHM allow following the state of health of the system and take the right decision at the right time to extend remaining useful life (RUL) of that system thanks mainly to prognostics. Applying PHM to a PEMFC stack is quite new and very few papers dealing with this subject can be found in literature. Of course a lot of works regarding PEMFC are available but considering them with a PHM point of view is a recent topic of research. [1] drew a state of the art of PHM of PEMFC and highlighted few challenges in that field. Performing prognostics on PEMFC stacks is one of them. Two papers intending to estimate the RUL of a stack are available until now. In [2], the authors propose a prognostic model based on an unscented Kalman filter in order to link the operating conditions to the degradation rate of the electrochemical active surface area. It shows interesting results according to the $\alpha$ performance metrics described in [3]. However, this prognostic is performed on a single cell (not a whole stack) and covers a short period of time $(300 \mathrm{~h})$, which is limited if we consider the expected 5,000 hours of lifetime needed for transportation applications or the 80,000 hours required for stationary ones. In [4], the voltage drop during the aging of a 5-cell stack is considered. A hybrid prognostics method is developed based on particle filtering. Three models are tested to estimate the global trend of voltage evolution through time and then predict the RUL. Promising results are showed. Yet, these models did not take into account any disturbance occurring during the stack aging, limiting the accuracy of predictions. This is the problem adressed in this paper.

This work intends to pursue and improve the work presented in [4] by including characterization-induced disturbances and voltage recoveries that can be observed during the stack aging. The main idea is to model the effects of disturbances and to integrate it into the prognostics scheme. To achieve that goal, PEMFC are first introduced, as well as their aging and the data used are presented in Section II. Then, the prognostics framework is introduced in Section III. A stack remaining useful life is estimated and the results are discussed. To prove the improvement with respect to our previous work, RUL estimates are performed on another stack and compared to our older predictions before concluding.

\section{Fuel Cell AGing}

\section{A. PEMFC System overview}

PEMFC technology uses hydrogen and oxygen to provide electricity, also producing heat and rejecting water. It is based 
on an electrochemical reaction that transforms chemical energy into electrical one (Figure 1). PEMFC can be used in several applications such as transportation, combined heat and power systems (micro-cogeneration) but also as a clean and efficient portable power converter for low power electronic devices. A PEMFC system is composed of a stack that converts the chemical energy into electrical one, surrounded by different ancillaries providing reactant to the system, collecting electricity or controlling the operating conditions. As the stack is the core of the system, the study is limited only to that element. The stack is an assembly of cells which are themselves made of different components. Descriptions of a stack and its components can be found in [5]. The total power provided by the stack is the sum of the power provided by the cells that are connected in series.

For prognostics purpose, it is interesting to know how the supplied power decrease as the stack ages. Due to multiphysics (thermodynamics, chemistry, etc.) and multiscale (from nanoscale particles to the system level) phenomena, the aging of a stack is difficult to observe and to understand. And it is even more difficult to model. Some mechanisms responsible for the performance degradation can be found in [6], [7], [8]. As an example, we can quote carbon corrosion, catalyst dissolution, membrane degradation, etc. Some degradation models are available [9]. However, they are not suited for prognostics as they take into account a limited number of degradation mechanisms or include parameters hard to measure, etc. Moreover, none of them is able to take into account the effects of disturbances appearing during the aging. In order to illustrate this last paragraph and to introduce what the disturbances introduced during the aging can be, the data available for prognostics are presented now and the authors' propositions on how to model the aging will be discussed after.

\section{B. Aging and characterizations data}

Data used for prognostics come from the aging of a 5-cell PEMFC stack. This stack was run under constant current load of $\mathrm{I}=60 \mathrm{~A}$ during approximately 1750 hours. Different measurements were made through the aging :

- load (to compare with imposed one);

- stack voltage evolutions through time;

- polarization curves;

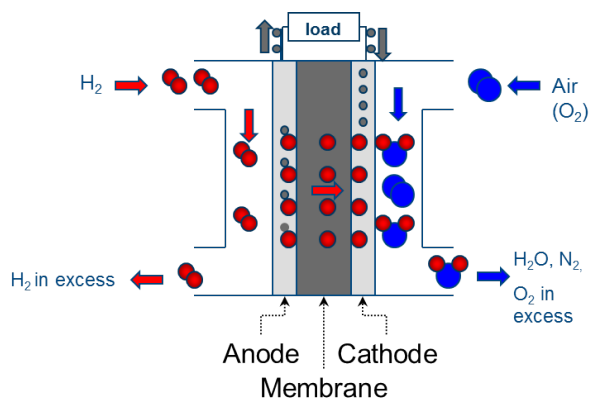

Fig. 1. PEMFC functioning principle

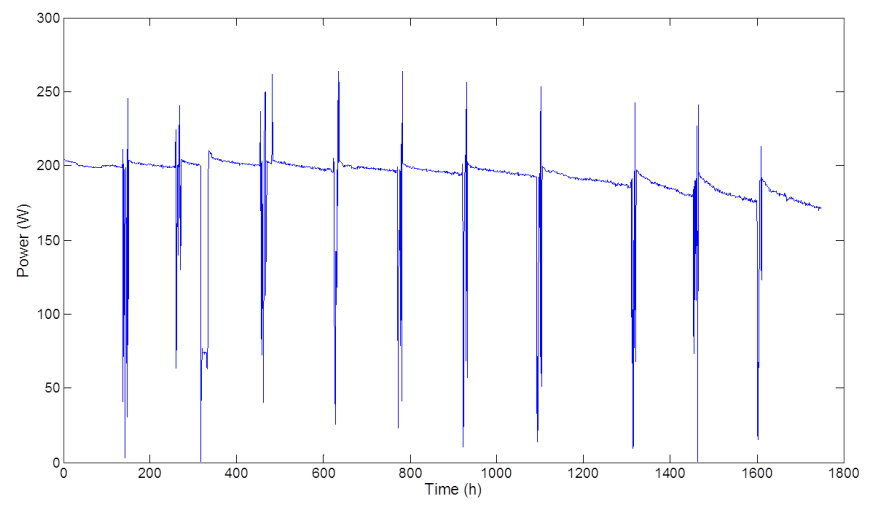

Fig. 2. PEMFC stack power degradation showing $\mathrm{v}$ and Rec

- electrochemical impedance spectroscopy (EIS).

For this study only the voltage and load measurements are used. However, it is important to mention the other characterization methods used because they are the ones that introduce the perturbations and recoveries observed on the voltage signals.

Indeed, for polarization curves and impedance measurements, current density variations are imposed to the stack. One can refer to [10] and [11] for more precisions on procedures. When the stack returns in its nominal functioning conditions after characterization, it recovers some performance, as it can be seen on Figures 2 and 3. No clear explanation can be given on what happened inside the stack. But, it can be assumed that returning to a nominal load demand after current variations re-homogenize the liquid and gas distributions within the stack, reset the operating conditions and allow thereby canceling what are called reversible degradations. Based on that, the recovery observed after characterizations is limited by irreversible degradations taking place into the stack. This distinction between reversible and irreversible degradation is discussed in [12].

Another interesting fact to observe is that the degradation seems to accentuate as the stack becomes older. It could be attributed to more degraded states for some components in the stack. That kind of acceleration has to be taken into account when constructing the aging model for prognostics.

\section{Aging modeling}

The operating conditions and load were kept constant, which allows us to formulate the hypothesis that the aging observed is time-effects. This imply that our prognostics model and all its parameters have only to be time-dependent.

1) Power degradation: To follow the aging of a stack, it is interesting to consider its power loss through time. The power is given by: Power $=$ Voltage $\mathrm{x}$ Load. In our case the load is constant so modeling the voltage drop is the similar as modeling the power drop. Only the model coefficients will change. Consequently, to describe the aging of the stack, three empirical models that can be adapted to both power and voltage degradations are set.

The first one aims at describing the global power degradation 


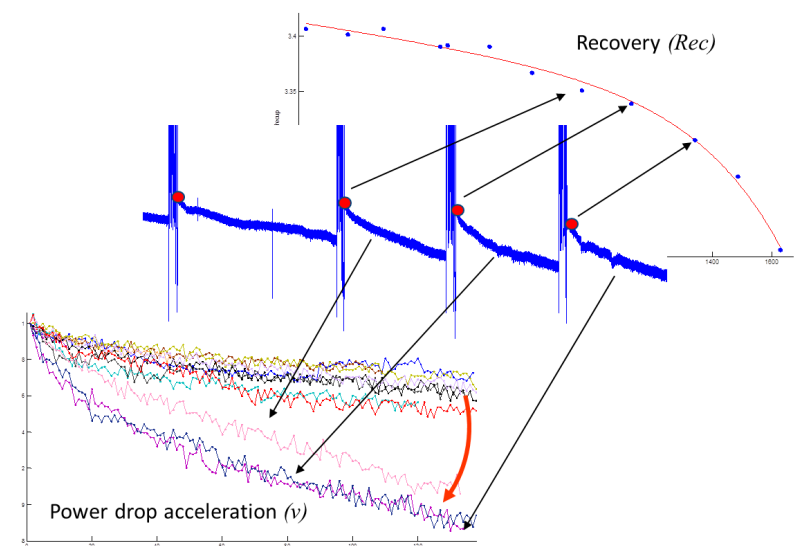

Fig. 3. $\quad v$ and Rec illustration

that can be observed between characterization phases. This model is composed by a logarithmic part and a linear one.

$$
x(t)=a_{1} \cdot \ln (t)-v \cdot t+c_{1}
$$

where $x(t)$ represents the power evolution through time, $a_{1}$ and $c_{1}$ coefficients to determine, and $v$ a coefficient driving the speed of degradation described after. The logarithmic part is supposed to model the transient phase and the linear one the steady decay period. This idea is based on observations but also according to [12] in which the authors suggest the presence of two functioning phases after a characterization.

2) Degradation's accelerations: However, by comparing the power drops between characterization phases, it can be seen that the power decreases faster and faster as the stack is aging and this should be taken into account. This is visible on left-hand bottom of Figure 3 in which all the parts between characterizations (normalized by their first value) are plotted together to be compared. It clearly shows that the power drops faster for the last parts. According to that, and assuming that $v$ evolves with time, a global trend for $v$ evolution has to be extracted. As $v$ is the slope of the linear part of the model, it can be obtained by calculating $d x / d t$ for certain number of point during the learning phase. This was done on different data sets and it enabled extracting a global trend for $v$ :

$$
v(t)=-a_{2} \cdot \exp \left(b_{2} \cdot t\right)+c_{2}
$$

where $v(t)$ is the coefficient from the power degradation model and $a_{2}, b_{2}$ and $c_{2}$ coefficients to determine. Considering the hypotheses of constant current demand and constant operating conditions, even if the starting point is completely different, this model for power aging shows a global trend close to the one proposed in [13] where a semi-empirical voltage degradation model for a stack used in a bus is set.

3) Recoveries: Then, the last thing to model is the power recovery observed after characterizations. This recovery is represented on Figure 3 by round dots. It is limited by the increasing in the irreversible degradation. As it still is very complicated to model irreversible degradations in a PEMFC, once again a global trend is extracted from different data sets. Consequently, the recovery follow this equation:

$$
\operatorname{Rec}(t)=a_{3} \cdot \exp \left(b_{3} . t\right)+c_{3} \cdot \exp \left(d_{3} . t\right)
$$

where $\operatorname{Rec}(t)$ is the recovery and $a_{3}, b_{3}, c_{3}$ and $d_{3}$ coefficients to determine.

\section{Prognostics Modeling}

These three models, equations (1-3), combined together make possible catching the behavior of the stack all along its lifetime.

A hybrid approach of prognostics is chosen. Indeed, it allows combining the data available with our empirical models. Moreover, to help choosing which prognostics method can be used, it can be noticed that the models built are nonexact, non-stationary, nonlinear and no Gaussian noise is introduced. These criteria are characteristics of a nonlinear Bayesian tracking problem [14], [15]. Different methods are available to solve that kind of problem, but only the particle filter solution was retained as it fits to our problem and shows good results in literature [16], [17], [18].

\section{A. Particle filtering framework}

A problem of tracking is defined by two equations [14], [15]. The first one, the state model, considers the evolution of a system state. The state noted $\left\{x_{k}, \mathrm{k} \in \mathbb{N}\right\}$ is going to evolve following

$$
x_{k}=f\left(x_{k-1}, \theta_{k}, \lambda_{k}\right)
$$

where $f$ is the transition function from the state $x_{k-1}$ to next state $x_{k}$, possibly nonlinear; $\theta_{k}$ is the vector of unknown parameters in the model and $\lambda_{k}$ an independent identically distributed (i.i.d.) noise (if existing). The tracking recursively estimates $x_{k}$ from measurements introduced by the second equation, the observation model $\left\{z_{k}, \mathrm{k} \in \mathbb{N}\right\}$

$$
z_{k}=h\left(x_{k}, \mu_{k}\right)
$$

where $h$ is the observation function and $\mu_{k}$ an i.i.d. noise.

The aim of the tracking problem is to recursively estimate, not directly the state of the system, but the probability distribution of the state at time $k$ by constructing the probability density function (pdf) $p\left(x_{k} \mid z_{1: k}\right)$. It is assumed that the initial pdf $p\left(x_{0} \mid z_{0}\right) \equiv p\left(x_{0}\right)$ of the state is available. $p\left(x_{k} \mid z_{1: k}\right)$ can be obtained recursively in two stages:

- prediction:

$$
p\left(x_{k} \mid z_{1: k-1}\right)=\int p\left(x_{k} \mid x_{k-1}\right) p\left(x_{k-1} \mid z_{k-1}\right) d x_{k-1}
$$

- update:

$$
p\left(x_{k} \mid z_{1: k}\right)=\frac{p\left(z_{k} \mid x_{k}\right) p\left(x_{k} \mid z_{1: k}\right)}{p\left(z_{k} \mid z_{1: k-1}\right)}
$$

This gives the optimal solution but in many cases it cannot be solved analytically. An approximate solution can be encountered by using the particle filtering framework.

The principle of particle filter is reminded. Particle filter is a Monte Carlo-based tool based on the Bayes' theorem. At 
the first stage $(k=1)$, the initial distribution $p\left(x_{0}\right)$ is split into $n$ samples, called particles. Then, the following steps are repeated until the end of the process.

1) Prediction Particles are propagated from state $k-1$ to state $k$ using the state model. A new pdf is obtained.

2) Update The coming of a new measurement $z_{k}$ allows to calculate the likelihood $p\left(z_{k} \mid x_{k}\right)$. This probability shows the degree of matching between the prediction and the measurement. Its calculation allows attributing weights at each particle according to the likelihood. Particles with higher weights represent the most probable states.

3) Re-sampling This stage appears to avoid a degeneracy of the filter. Indeed after several iterations, the particle with low weights become too numerous altering the prediction step. Their are different kind of re-sampling strategies but the principle remains the same. Particles with the lower weights (compared to a chosen limit weight) are eliminated whereas the ones with higher weights are duplicated.

\section{B. Architecture including perturbations}

The operating conditions remaining constant, perturbations are only induced by characterization. Their occurrence is planned before the beginning of aging tests. Consequently, this planning can be added to the prognostics structure (Table I). As for any Bayesian tracking problem a state equation $x_{k}$ and a measurement equation $z_{k}$ have to be defined. Before doing, the architecture defined should be explained. Considering the problem and the number of unknown coefficients in our models, a structure with three particle filters working in parallel is defined. The first one estimates the power aging, the second one the coefficient $v$ and the last one estimates the recovery Rec after characterization. With this structure each filter estimates its attributed parameters but the three of them are always synchronized on the same time step.

When the date of a characterization is detected, it means that a power recovery is going to happen. To account for that, the power particles (filter 1) values is updated with the particles values coming from the filter dedicated to the recovery estimation (filter 2). As a characterization also represents the beginning of a faster power drop, the power model is updated with the last values of $v$ coming from the filter 2 . This architecture is drawn on Figure 4.

The use of three filters implies three state models built thanks to equations (1-3). There are defined as:

$$
\begin{gathered}
x_{k}=-a_{1} \cdot \ln (1 / k+1)+v_{k} \cdot d t+x_{k-1} \\
v_{k}=-a_{2} \cdot \exp \left(b_{2} \cdot k\right) \cdot\left(1-\exp \left(-b_{2}\right)\right)+v_{k-1} \\
R e c_{k}=a_{3} \cdot \exp \left(b_{3} \cdot k \cdot d t\right) \cdot\left(1-\exp \left(-b_{3}\right)\right) \\
+c_{3} \cdot \exp \left(d_{3} \cdot k \cdot d t\right) \cdot\left(1-\exp \left(-d_{3}\right)\right)+R e c_{k-1}
\end{gathered}
$$

where $d t$ is the time step between two measurements (here 1 hour). Regarding the measurement model, it simply contains the measurements available during the learning phase.

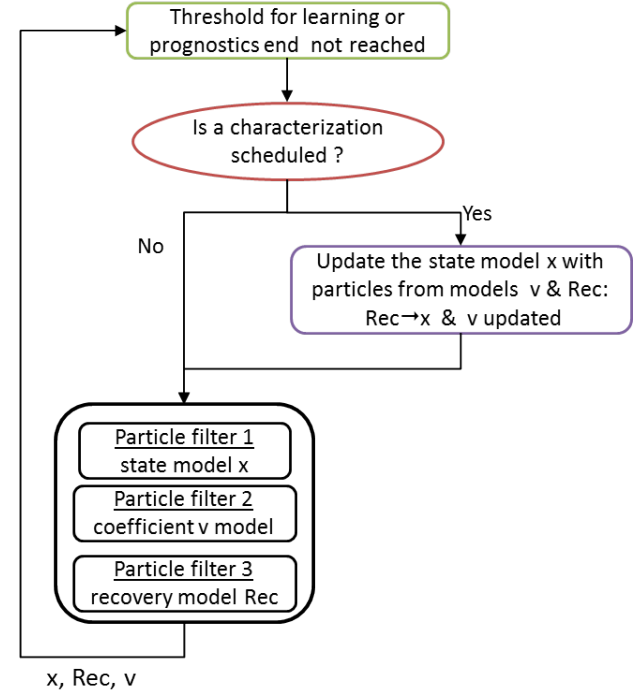

Fig. 4. Architecture of the prognostics structure

\section{Defining the thresholds}

Failure thresholds for fuel cells are defined according to a percentage of power drop compared to the initial one. According to the US Department of Energy [19], the degradation should not exceed $10 \%$ of the initial power on a $2500 \mathrm{~h}$ life duration. The stack technology available for experiments always fails to meets these requirement. Also our global loss of power for the stack is $16,24 \%$ on a $1750 \mathrm{~h}$ lifetime. Consequently, $16 \%$ is chosen as a failure threshold for RUL predictions.

\section{EXPERIMENTS AND DISCUSSION}

This section intends to show the performance of the previously defined prognostics framework.

\section{A. Model fitting and estimates}

Regarding the number of unknown coefficients that can be learned by the filters, the learning data set should contain at least 4 characterization phases. Consequently, the earlier end for the learning phase cannot be smaller than 500 hours. The predictions are made for learning phases ranging from 500 to 1700 hours by increasing the length of the training set by 50 hours each time.

In order to estimate the remaining useful life, the power degradation is estimated. One example of this estimation is showed on Figure 5. It can be seen that before 1100 hours, the framework catches pretty well the degradation of the power although the logarithmic part of the model seems to be ignored by the estimation. After 1100 hours, the recovery function looks like being over-estimated and the error on the recovery after the last four characterizations begins to increase. However, the power drop driven by the coefficient $v$ seems to values close the reality. Once again the logarithmic part influence, driven by coefficient $a_{1}$, is absent. It could be interesting to find if this coefficient follows a particular trend during aging and model it. These comments show that some, maybe all, models have to be improved. 
TABLE I

CHARACTERIZATIONS SCHEDULING

\begin{tabular}{|c|c|c|c|c|c|c|c|c|c|c|c|c|}
\hline Characterization number & 1 & 2 & 3 & 4 & 5 & 6 & 7 & 8 & 9 & 10 & 11 & 12 \\
\hline Date (hours) & 137 & 259 & 317 & 455 & 480 & 624 & 770 & 921 & 1091 & 1310 & 1453 & 1600 \\
\hline
\end{tabular}

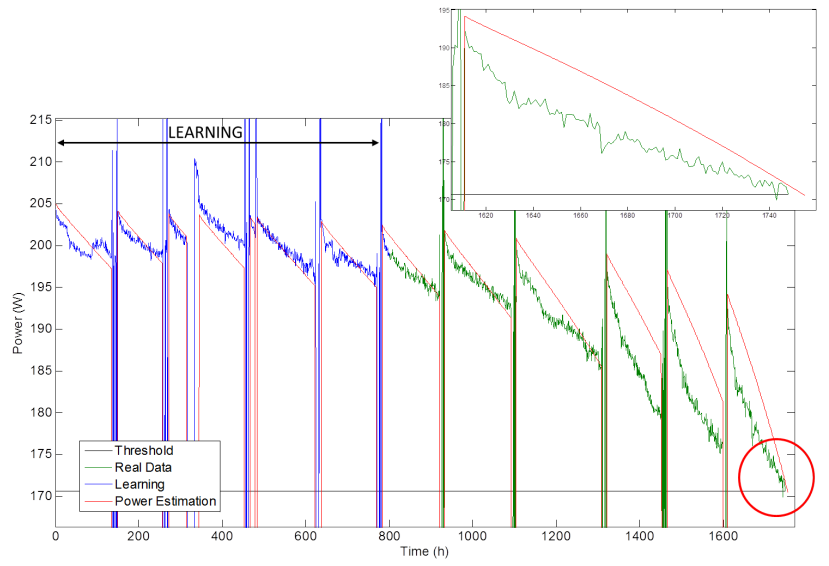

Fig. 5. Predicted degradation of the power through time illustrated with a training of $800 \mathrm{~h}$

\section{B. Uncertainty estimation}

Series of tests on power degradation estimates showed that particles of the filters tend to converge to the same state value. As a consequence, instead of obtaining a final distribution of the RUL, only one value is given and the uncertainty coming with this estimation remains unknown.

To avoid this problem, the uncertainty introduced by the prognostics framework has to be evaluated. To do so the prognostics framework is launched 100 times with exactly the same initialization and the same duration of learning. This process is illustrated on Figure 6 on which the distribution of the results is presented for a 600 hours long training. It can be seen that the results are normally dispersed and that all the predictions are contained in a small interval. This kind of experiment allows to state that the prognostics framework gives estimates contained in a range of 20 hours. By assuming that these estimates are normally distributed (as in the example), we can say that our framework gives prediction with an uncertainty of \pm 10 hours.

\section{RUL estimates}

Although, the degradation estimation remains imperfect, the RUL estimates are quite convincing (Figure 7). All the predictions are located in a $99 \%$ interval around the actual RUL, which represent \pm 17.48 hours of the actual RUL. If we add the uncertainty estimated above, it means that our framework can give results precise at \pm 27.48 hours.

\section{Discussion}

The results presented here are very promising. But the framework still suffers from a few drawbacks. First, the threshold chosen as the end of life criterion is almost the end of the data set. But some trials with thresholds that are located

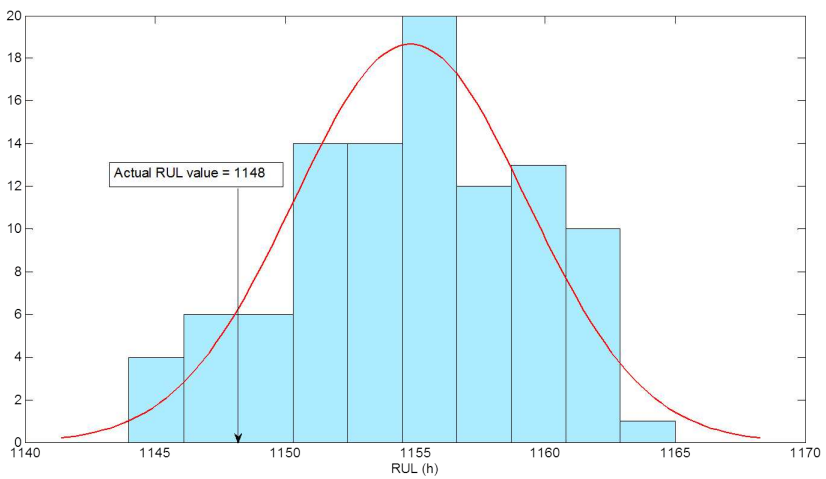

Fig. 6. Uncertainty associated to the RUL estimates at $\mathrm{t}=600 \mathrm{~h}$

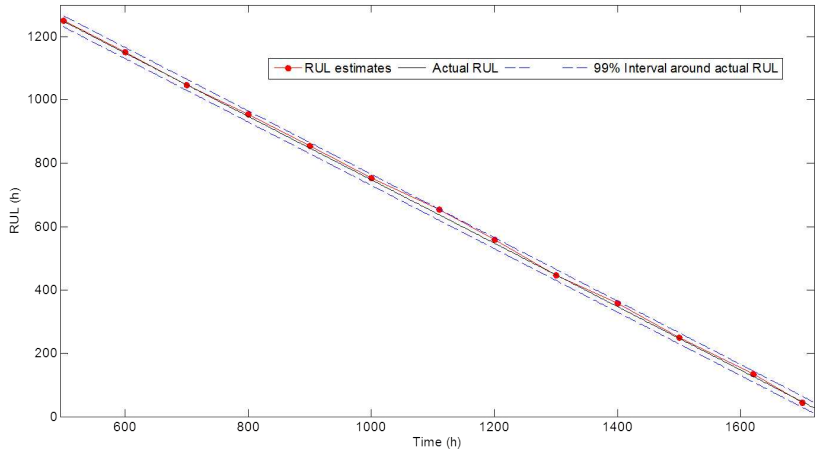

Fig. 7. RUL predictions

between $9.5 \%$ and $14.4 \%$ of the initial power did not show so good results. One explanation has already be mentioned earlier, namely the weakness of some parts of the models. Further analysis on PEMFC degradation may help to refine these models. Another explanation could be the initialization of the prognostics framework, if the filters are not given the right initial distributions they will not converge to the right parameters values. However, the good power estimations until 1100 hours do not seem to confirm that hypothesis.

\section{E. Comparison with the original framework}

To compare the improvement with the orignal framework presented in [4], a new serie of tests is performed on older data set refered as "Fuel cell 2" in our previous paper. These data also come from a 5-cell stack aging under a constant current (of 70A this time) during approximately 1000 hours. Here, as shown on Figure 8, we only compare the RUL estimates. As we can see on the upper part of the figure, the accuracy improvement on the RUL estimate itself is not so impressive, even if the new predictions are a little closer the actual RUL value. But it can be noticed that the new framework gives less late predictions. 

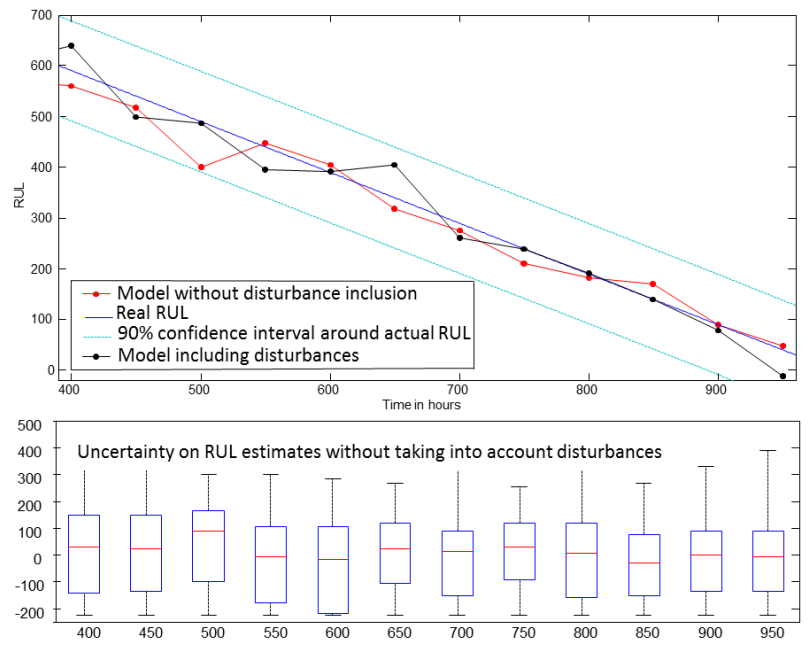

Fig. 8. Comparison of RUL estimates by taking into account or not taking into account disturbances \& Uncertainty associated with the original framework

However, the major improvement can be seen in the uncertainty coming with the predictions. As illustrated on the bottom part of the figure, previous RUL estimates were given with an accuracy of \pm 90 hours and with an associated uncertainty ranging from 112 to 388 hours in the worst case. With the modeling of recoveries and power drop acceleration but also the improvement of the prognostics framework, the accuracy is now of \pm 40 with an uncertainty of \pm 10 hours. From a decision making point of view, these last results are far more interesting. They also clearly show that the more phenomena are modeled the more accurate are our results.

\section{CONCLuSion}

This paper presents a new framework for remaining useful life predictions of a PEMFC stack. A previous work [4] showed that characterization-induced perturbations cannot be ignored to predict the future behavior of the stack. By combining a global model for power degradation regularly, a model that reflects the acceleration of the drop with time and a last one that takes into account power recoveries, a more effective prognostics framework is developed. Even if, the behavior prediction is still not perfect, RUL estimates are quite encouraging as the error going with the predictions is smaller than 28 hours for a global lifetime of 1748 hours. A next step to that work will be first the reinforcement of the models, to be able then to start integrating variable operating conditions.

\section{ACKNOWLEDGMENT}

This work has been supported by the ANR project PROPICE (ANR-12-PRGE-0001) and by the Labex ACTION project (contract "ANR-11-LABX-01-01") both funded by the French National Research Agency.

\section{REFERENCES}

[1] M. Jouin, R. Gouriveau, D. Hissel, M.-C. Péra, and N. Zerhouni, "Prognostics and health management of PEMFC state of the art and remaining challenges," International Journal of Hydrogen Energy, vol. 38, no. 35, pp. 15307 - 15317, 2013.

[2] X. Zhang and P. Pisu, "An unscented kalman filter based approach for the health-monitoring and prognostics of a polymer electrolyte membrane fuel cell," in Proceedings of the annual conference of the prognostics and health management society, 2012.

[3] A. Saxena, J. Celaya, B. Saha, S. Saha, and K. Goebel, "On applying the prognostic performance metrics," in Proceedings of the annual conference of the prognostics and health management society, 2009.

[4] M. Jouin, R. Gouriveau, D. Hissel, M.-C. Péra, and N. Zerhouni, "Prognostics of PEM fuel cell in a particle filtering framework," International Journal of Hydrogen Energy, vol. 39, no. 1, pp. 481 - 494, 2014.

[5] J. Larminie, A. Dicks, and M. S. McDonald, Fuel cell systems explained. Wiley Chichester, 2003, vol. 2, no. 1.

[6] W. Schmittinger and A. Vahidi, "A review of the main parameters influencing long-term performance and durability of PEM fuel cells," Journal of Power Sources, vol. 180, no. 1, pp. 1 - 14, 2008.

[7] J. Wu, X. Z. Yuan, J. J. Martin, H. Wang, J. Zhang, J. Shen, S. Wu, and W. Merida, "A review of PEM fuel cell durability: Degradation mechanisms and mitigation strategies," Journal of Power Sources, vol. 184 , no. 1 , pp. $104-119,2008$.

[8] R. Borup, J. Meyers, B. Pivovar, Y. S. Kim, R. Mukundan, D. Garland, N.and Myers, F. Wilson, M.and Garzon, D. Wood et al., "Scientific aspects of polymer electrolyte fuel cell durability and degradation," Chemical reviews, vol. 107, no. 10, pp. 3904-3951, 2007.

[9] A. Franco, "Modelling and analysis of degradation phenomena in pemfc." Woodhead, 2012.

[10] FCTESQA. (2009) Pefc power stack performance testing procedure measuring voltage and power as function of current density polarisation curve test method. [Online]. Available: http://iet.jrc.ec.europa.eu/fuel-cells/sites/fuelcells/files/files/documents/Polarisation_Curve_TestProcedure_ST_53.pdf

[11] A. Lasia, Electrochemical Impedance Spectroscopy and its Applications, B. E. Conway, J. O. Bockris, and R. E. White, Eds. Springer US, 1999, vol. 32 .

[12] S. Kundu, M. Fowler, L. C. Simon, and R. Abouatallah, "Reversible and irreversible degradation in fuel cells during open circuit voltage durability testing," Journal of Power Sources, vol. 182, no. 1, pp. 254 $-258,2008$.

[13] L. Lu, M. Ouyang, H. Huang, P. Pei, and F. Yang, "A semi-empirical voltage degradation model for a low-pressure proton exchange membrane fuel cell stack under bus city driving cycles," Journal of Power Sources, vol. 164, no. 1, pp. $306-314,2007$.

[14] A. Doucet, N. de Freitas, N. Gordon, and A. Smith, Sequential Monte Carlo Methods in Practice, ser. Information Science and Statistics. Springer, 2010

[15] M. S. Arulampalam, S. Maskell, N. Gordon, and T. Clapp, "A tutorial on particle filters for online nonlinear/non-gaussian bayesian tracking," Signal Processing, IEEE Transactions on, vol. 50, no. 2, pp. 174-188, 2002.

[16] D. An, J.-H. Choi, and N. H. Kim, "A tutorial for model-based prognostics algorithms based on matlab code," in Proceedings of the annual conference of the prognostics and health management society, 2012.

[17] K. Goebel, B. Saha, A. Saxena, J. Celaya, and J. Christophersen, "Prognostics in battery health management," Instrumentation Measurement Magazine, IEEE, vol. 11, no. 4, pp. 33-40, 2008.

[18] Y. Xing, E. W. Ma, K.-L. Tsui, and M. Pecht, "An ensemble model for predicting the remaining useful performance of lithium-ion batteries," Microelectronics Reliability, vol. 53, no. 6, pp. 811 - 820, 2013.

[19] U. D. of Energy. (2011) The department of energy hydrogen and fuel cells program plan. [Online]. Available: http://www.hydrogen.energy.gov/roadmaps_vision.html 